\title{
STATISTICAL MECHANICS OF SOME CO-OPERATIVE PHENOMENA
}

\author{
By C. DOMB \\ Pembroke College, Cambridge*
}

\section{$\mathrm{T}$} HERE are a number of co-operative phenomena associated with specific heat singularities for which the echnical mathematical problems are formally sjnilar. These include the Ising model of a ferromagnetic, the theory of regular binary solid solutions, and the order-disorder transitions in allofs. Many approximations have been devised for treating these problems, and as a typical example we may quote the Bethe approximation ${ }^{1}$. It was at first considered that these approximations provided a satisfactory account of the thermodynamics of the problems. Recently, however, the exact solution of a particular problem by Onsager ${ }^{2,3}$ has shown that the standard approximations are unreliable in the neighbourhood of the singularity, and has focused attention on exact solutions.

It is clarifying to consider the above problems collectively, and in a recent paper ${ }^{4}$ it has been shown that, if only nearest neighbour interactions are taken into account, the thermodynamic properties are completely determined by a function $\Lambda(\mu, z)$ of two positive variables $\mu$ and $z$. For the ferromagnetic problem, the free energy is given (except for a zeroorder term) by

$$
F=-n k T\left[\log \Lambda(\mu, z)-\frac{1}{2} \log \mu\right],
$$

where $z=\exp (-\varepsilon / k T), \mu=\exp (-2 m H / k T)$, and $\varepsilon$ is an interaction energy. In the regular solution and order-disorder problems, the corresponding free energy is

$$
F^{\prime}=-n k T\left[\log \Lambda(\mu, z)-\alpha_{1} \log \mu\right],
$$

where $\mu$ now depends on $\alpha_{1}$, the mole fraction of constituent 1 , and is determined by the relation

$$
\mu \Lambda_{\mu} / \Lambda=\alpha_{1} \text {. }
$$

It is easy to establish the relation

$$
\mu^{-1 / 2} \Lambda(\mu, z)=\mu^{1 / 2} \Lambda(1 / \mu, z),
$$

and this means that only values of $\mu$ from 0 to $I$ need be considered. The ferromagnetic and regular solution problems correspond to values of $z$ between 0 and 1 , and we shall discuss them shortly. The order-disorder and anti-ferromagnetic problems cor-

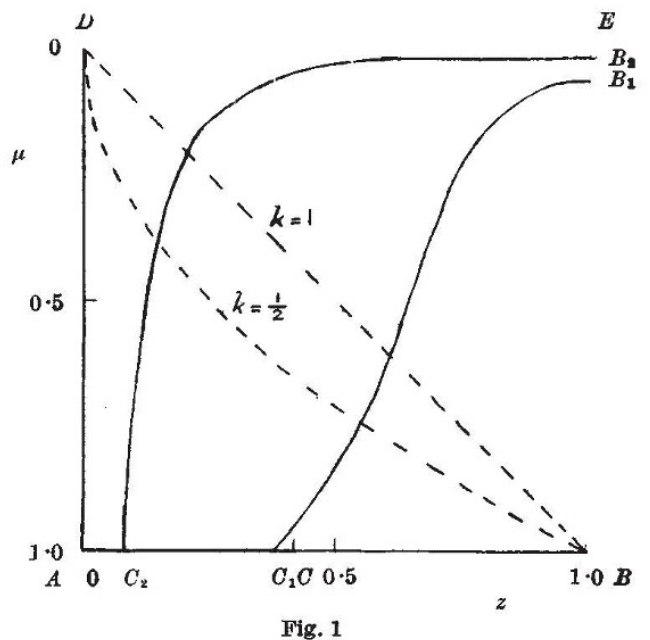

respond to values of $z^{-1}$ between 0 and 1 , and we shall not consider them further here.

It is well known that a one-dimensional system does not exhibit sharp phase transitions ${ }^{5}$, and therefore, to obtain information of interest, we must pro. ceed to a two-dimensional system. In the paper referred to above ${ }^{4}$, a generalization of some earlier work of Kramers and Wannier ${ }^{6}$ shows that $\Lambda(\mu, z)$ is the largest eigenvalue of an infinite matrix of simple characteristic structure. A discussion of the properties of $\Lambda(\mu, z)$, and hence of $F$ and $F^{\prime}$, is best undertaken with the aid of Fig. 1, which represents the $(\mu, z)$ plane. Onsager's solution applies to the line $A B$, and starting at $A$ (corresponding to $T=0$ ) a power series may be derived, of which the first terms are the following :

$$
\begin{aligned}
& \Lambda(1, z)=\lambda(z)=1+z^{4}+2 z^{6}+5 z^{8}+ \\
& 14 z^{10}+44 z^{12}+152 z^{14}+\ldots
\end{aligned}
$$

This series is valid up to the Curie point $C(z=0 \cdot 414)$ and gives rise to a logarithmic infinity in $\lambda_{z z}$ (specific heat singularity) at $C$. The following generalization of this series for values of $\mu$ other than 1 has been obtained :

$$
\begin{gathered}
\Lambda(\mu, z)=1+\mu z^{4}+2 \mu^{2} z^{6}+\left(-2 \mu^{2}+6 \mu^{3}+\mu^{4}\right) z^{8}+ \\
\left(-14 \mu^{3}+18 \mu^{4}+8 \mu^{5}+2 \mu^{6}\right) z^{10}+ \\
\left(8 \mu^{3}-77 \mu^{4}+44 \mu^{5}+40 \mu^{6}+22 \mu^{7}+\right. \\
\left.6 \mu^{8}+\mu^{9}\right) z^{12}+\left(98 \mu^{4}-370 \mu^{5}+40 \mu^{6}+138 \mu^{7}+\right. \\
\left.134 \mu^{8}+72 \mu^{0}+30 \mu^{10}+8 \mu^{11}+2 \mu^{12}\right) z^{14}+\ldots
\end{gathered}
$$

Substitution of $\mu=1$ in (6) gives back (5), so this series seems to be valid up to $\mu=1$, and there is no discontinuity in $\Lambda$ at $\mu=1$. On the other hand, we can deduce from (4) that $\Lambda_{\mu} / \Lambda=1 / 2$ at $\mu=1$, whereas from (6) $\Lambda_{\mu} / \Lambda \rightarrow \beta(z)$ as $\mu \rightarrow 1$, where

$\lambda(z) \beta(z)=z^{4}+4 z^{6}+18 z^{8}+82 z^{10}+387 z^{12}+$

$$
1880 z^{14}+\ldots .
$$

Thus there is a discontinuity in $\Lambda_{\mu}$ as $\mu \rightarrow 1$ in the neighbourhood of $A$, which one would expect to persist as far as $C$. High-temperature expansions starting at $B$ indicate (as one would expect) that this discontinuity does not persist beyond $C$.

Onsager's solution for $\mu=1$ corresponds to a ferromagnetic in the absence of a magnetic field, and values of $\mu<1$ correspond to the presence of a magnetic field. The magnetization is given by $-\partial F / \partial H$ in (1), and hence by

$$
I / I_{\infty}=1-2 \mu \Lambda_{\mu} / \Lambda \text {. }
$$

Thus the discontinuity in $\Lambda_{\mu}$ gives rise to a spontaneous magnetization

$$
I_{0} / I_{\infty}=1-2 \beta(z)
$$

which is shown as a function of $z$ in Fig. 2. In the presence of a constant magnetic field, the thermodynamic properties are obtained by following a curve of the form $\mu=z^{k}$, where $k$ is a measure of the strength of the field. A study of the terms of (6) for values of $\mu<1$ indicates a completely different behaviour from that for $\mu=1$, caused by the large negative term in the coefficient polynomials in $\mu$. This is shown by the accompanying table.

For $\mu=1$, the terms are all positive and steadily increase, and this means a singularity on the real * Now at the Clarendon Laboratory, oxford. 


\begin{tabular}{|c|c|c|c|c|c|c|}
\hline Coefficient of : & $z^{4}$ & $z^{8}$ & $z^{8}$ & $z^{10}$ & $z^{12}$ & $z^{14}$ \\
\hline$\mu=1$ & 1 & 2 & 5 & 14 & 44 & 152 \\
0.9 & $0 \cdot 9$ & 1.62 & $3 \cdot 41$ & $7 \cdot 39$ & 16.04 & $32 \cdot 19$ \\
0.8 & $0 \cdot 8$ & 1.28 & $2 \cdot 20$ & $3 \cdot 35$ & $3 \cdot 21$ & $-5 \cdot 48$ \\
0.7 & $0 \cdot 7$ & 0.98 & $1 \cdot 32$ & $1 \cdot 10$ & $-1 \cdot 44$ & $-10 \cdot 92$ \\
\hline
\end{tabular}

axis $^{2}$. On the other hand, for $\mu=0.7$ and 0.8 , the terms cease to increase steadily, and there are strong indications that for any $\mu<1$ the coefficients are not consistent in sign beyond some point. This leads one to conjecture that the specific heat transition becomes continuous in the presence of a magnetic field, which is also the result given by the Heisenberg theory.



The exact theory of binary solid solutions was considered by Lassetre and Howe ${ }^{8}$, and we shall find many of their conclusions here substantiated. Considering the thermodynamics of a solid solution of fixed constitution, we must follow a path in the $(\mu, z)$ plane given by (3). When $\alpha_{1}=1 / 2$, this is the path $A B$ in Fig. 1. When $\alpha_{1}$ is different from $1 / 2$, however, a completely different type of path results. When $z<0.414$, the general behaviour of $\mu \Lambda_{\mu} / \Lambda$ as a function of $\mu$ is similar to the curve in Fig. 3 (for $z=0.4$ ). Its value steadily increases from zero at $\mu=0$ to $\beta(z)$ at $\mu=1$, and there is a jump to $1 / 2$ at $\mu=1$. This discontinuity arises for an infinite lattice, and would be a very rapid continuous change for a large finite lattice. Hence if $\beta(z)<\alpha_{1}<1 / 2$, the relation (3) can only be satisfied on the line $A B$. For





Fig. 4. $(z=0 \cdot 4)$

very high temperatures it can be shown that (3) leads to $\mu=\alpha_{1} /\left(l-\alpha_{1}\right)$. Thus for any value of $\alpha_{1}$ different from $1 / 2$, the path given by (3) moves along $A C$ until $\beta(z)=\alpha_{1}$, and then it turns into the plane, cutting $B E$ in a point $\mu=\alpha_{1} /\left(1-\alpha_{1}\right)$. Typical paths are $A C_{1} B_{1}, A C_{2} B_{2}$ in Fig. 1.

Clearly a transition takes place at points such as $C_{1}$, and we shall interpret the portion $A C_{1}$ as corresponding to a separation into two phases, and the portion $C_{1} B_{1}$ as solution. This interpretation is borne out by the curve of free energy as a function of concentration, which for $z<0.414$ is of the form shown in Fig. 4. The point on $A C$ in Fig. 1 corresponding to the value of $z$ considered may be regarded as a mixture of two phases at $Y$ in Fig. 4, phase $I$ being a solution of $\beta(z)$ of substance 1 in substance 2, and phase II a solution of $\beta(z)$ of substance 2 in substance $1 . \beta(z)$ is thus the maximum concentration which can exist in solution at the given temperature, and is, in fact, the solubility curve for the given substances; it is shown in Fig. 2. As we move along $A C_{1}$, the proportion of phase II gets smaller, until at $C_{1}$ it disappears.

The nature of the transition at $C_{1}$ can be determined from (2) and (3). There is a discontinuity in $\mu_{z}$ at $C_{1}$ given by

$$
\operatorname{Lt}_{\mu \rightarrow 1} \frac{\alpha_{1} \Lambda_{z}-\mu \Lambda_{\mu z}}{\mu \Lambda_{\mu \mu}+\left(1-\alpha_{1}\right) \Lambda_{\mu}} .
$$

On the other hand, $\left(F^{\prime} / T\right)_{\mu}=0$, so that $E=$ $n \varepsilon z \Lambda_{z} / \Lambda$, and this does not involve $\mu_{z}$. Thus the energy is continuous, but the specific heat has a discontinuity given by

$$
n k(z \log z)^{2} \underset{\mu \rightarrow 1}{L t}\left[\mu_{z} \frac{\partial}{\partial_{\mu}}\left(\Lambda_{z} / \Lambda\right)\right],
$$

so that the transition is second order.

It does not seem unreasonable to assume that, with the exception of the detailed behaviour at the singularity $C$, the above discussion should be qualitatively applicable to a three-dimensional model.

I am indebted to Prof. M. H. L. Pryce and Dr. G. S. Rushbrooke for helpful discussion and to the Department of Scientific and Industrial Research for a senior research award.

${ }^{1}$ Bethe, H. A., Proc. Roy. Soc., A, 150, 552 (1935).

2 Onsager, L., Phys. Rev., 65, 117 (1944).

${ }^{3}$ Wannier, G. H., Rev. Mod. Phys., 17, 50 (1945).

4 Domb, C., Proc. Roy. Soc., 196, 36 (1949).

${ }^{5}$ Rushbrooke, G. S., and Ursell, H. D., Proc. Camb. Phil. Soc., 44 263 (1948).

- Kramers, H. A., and Wannier, G. H., Phys. Rev., 60, 252, 263 (1941).

7 Dienes, P., "The Taylor Series", chap. 14 (Oxford, 1931).

${ }^{8}$ Lassetre, E. N., and Howe, J. P., J. Chem. Phys., 9, 747, 801 (1941). 